\title{
Oscillations in a maturation model of blood cell production
}

\author{
Ivana Drobnjak and A. C. Fowler \\ Mathematical Institute, Oxford University \\ 24-29 St Giles', Oxford OX1 3LB, England \\ Michael C. Mackey \\ Departments of Physiology, Physics and Mathematics, and \\ Centre for Nonlinear Dynamics, \\ McGill University, Montreal, Quebec \\ Canada
}

October 30, 2018

\section{Introduction}

A number of hematological diseases are characterised by oscillations in the circulating density of various types of blood cells. These include chronic myelogenous leukaemia $(\mathrm{CML})$, cyclical neutropenia $(\mathrm{CN})$, polycythemia vera $(\mathrm{PV})$ and aplastic anaemia (AA). Examples of blood cell counts for CML and CN are shown in figures 1 and 2

A review of the clinical data, and a discussion of possible mechanisms for the oscillations, is given by Haurie et al. (1998). These mechanisms focus on the rôle of negative feedback control on proliferation and differentiation of blood cells within the bone marrow, together with time delays due to cell cycling and maturation. There are consequently a number of different ways in which oscillations can occur, and one object of mathematical modelling of blood cell development is to understand which of these effects may be responsible for the oscillations which are seen.

Blood cells are produced through a process of differentiation from primitive stem cells in the bone marrow. These pluripotential stem cells begin to develop along one of several different cell lineages, forming blast cells which eventually develop through a number of different stages to form the various different kinds of blood cells. The most numerous are the red blood cells, or erythrocytes, whose normal density in the blood is about $5 \times 10^{6}$ cells $\mu \mathrm{l}^{-1}$. Their primary function is in transporting oxygen to the tissues. Platelets are formed by the fragmentation of megakaryocytes, which develop in the bone marrow. Platelets are present at levels of $5 \times 10^{5}$ cells $_{\mu \mathrm{l}^{-1}}$, and their function is in blood clotting. Finally, there are a number of different white blood cells, the most common of which are neutrophils (5000 cells $\left.\mu \mathrm{l}^{-1}\right)$ and lymphocytes 


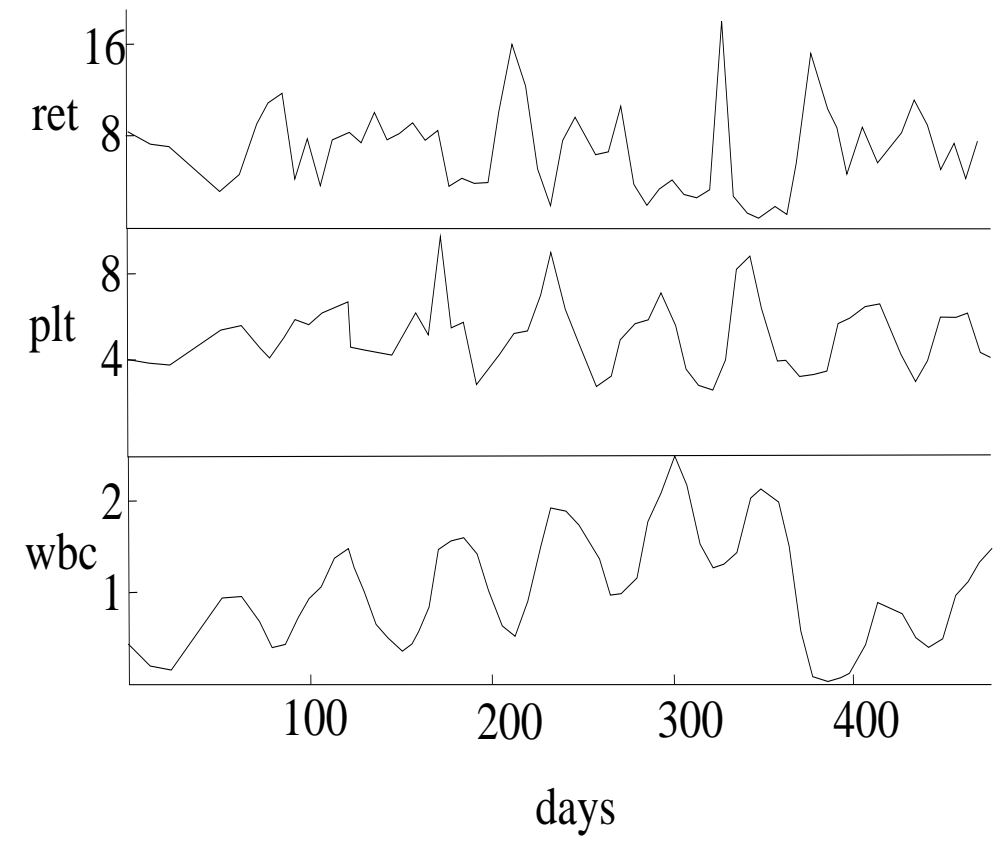

Figure 1: Oscillations in white blood cell, platelet and reticulocyte numbers in a patient with chronic myelogenous leukaemia. The units are white blood cells, $10^{5}$ cells $\mu \mathrm{l}^{-1}$; platelets, $10^{5}$ cells $\mu \mathrm{l}^{-1}$; reticulocytes, $10^{4}$ cells $\mu \mathrm{l}^{-1}$. Redrawn from Chikkappa et al. (1976).

(2000 cells $\mu \mathrm{l}^{-1}$ ), which form constituent parts of the immune system. The normal levels of these cells are controlled by a number of mechanisms, and excess or deficit of the various cell types defines certain kinds of disease; for example, anaemia refers to a low red blood cell count, below $4 \times 10^{6}$ cells $\mu \mathrm{l}^{-1}$.

There are a number of features in figures 1 and 2 which are of note. In CML, there are regular oscillations in white blood cell counts with a long period ranging from 40 to 80 days (Fortin and Mackey (1999)). The other cell lines (platelets and reticulocytes, i. e. erythrocyte precursors) also oscillate in a similar fashion (figure 1] does not show this well: see Fortin and Mackey (1999) for other examples).

A similar observation is true of cyclical neutropenia. Oscillation periods are of order 20 days, during which there is a marked collapse of the neutrophil count to vanishingly low levels (Dale and Hammond 1988, Guerry et al. 1973). Other cell types oscillate, but only the neutrophils appear to oscillate fairly regularly: oscillations in other cell types (e.g., red blood cells, platelets, reticulocytes and lymphocytes) are marked by irregularity and high frequency 'noise' (Guerry et al. 1973). This latter feature is well illustrated in figure 2,

The purpose of the present paper is throw some light on these observations by the study of a model of blood cell proliferation and differentiation. This model is similar to those of previous authors, particularly that of Mackey and Rudnicki (1994), and describes the stem cell and developing (blast) cell populations as functions of time, age (time through the proliferative cell cycle), and maturation (stage in the 

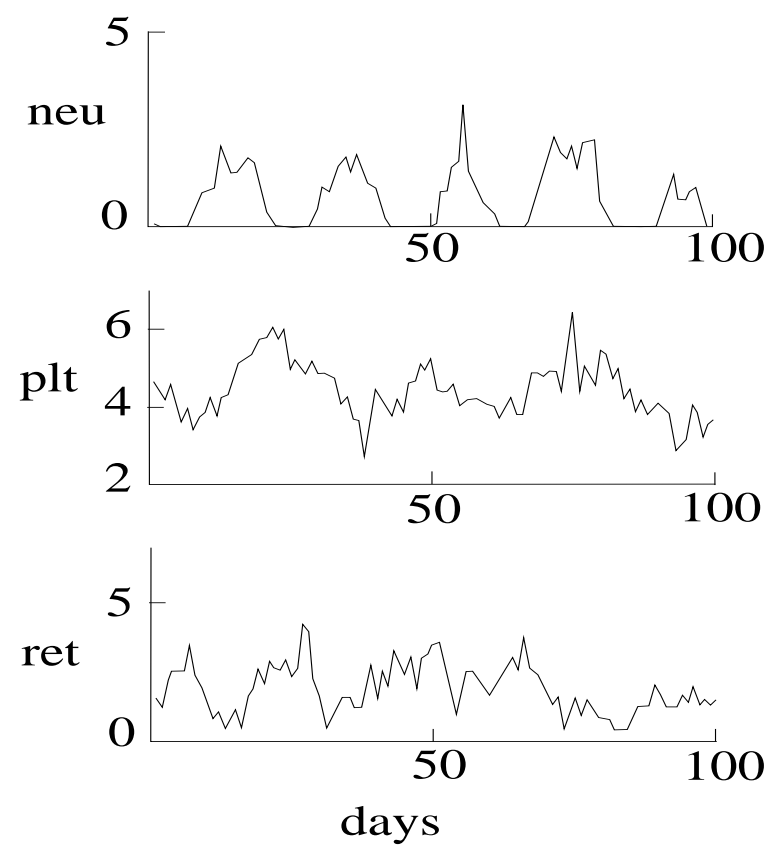

Figure 2: Oscillations in neutrophils, platelet and reticulocyte numbers in a patient with cyclical neutropenia. The units are neutrophils, $10^{3}$ cells $\mu \mathrm{l}^{-1}$; platelets, $10^{5}$ cells $\mu \mathrm{l}^{-1}$; reticulocytes, $10^{4}$ cells $\mu \mathrm{l}^{-1}$. Redrawn from Haurie et al. (1998).

differentiation process). Fokas et al. (1991) describe a model with discrete generations in the development of blast cells, while Mackey and Rudnicki (1994) develop a corresponding continuous model (i. e., developmental stage is a continuous variable).

In this paper we use a continuous model to describe the development of a single cell lineage following the committal of stem cells. Three separate controls are implemented in the model, namely the proliferative control of stem cells, the proliferative control of developing blast cells, and the peripheral control of stem cell committal by circulating blood cell density. We show that variation of parameters in all three control systems can cause oscillations, and that the characters of these oscillations are very different. This allows us some potential insight into the mechanisms that may be operative in some of these dynamic blood diseases.

\section{A model of maturation of blood cell production}

The basic model is similar to that described by Mackey and Rudnicki (1994). It has been analysed in various versions by Rey and Mackey (1993), Crabb et al. (1996) and Mackey and Rudnicki (1999). A particular feature of these models was the assumption of zero maturation rate at maturation state zero. In our formulation of the model, we do not make this assumption.

We consider all cell lineages to consist of populations of two types, proliferative and resting phase. These are denoted $p$ and $n$, respectively, and are functions of age 
$a$ (time since the inception of the proliferative cell cycle) and maturation $m$ (degree of maturation, measured in maturation units (mat), which could be, for example, cell generation number). Also, $p$ and $n$ are functions of time $t$. Thus, we have $p=p(t, m, a)$ and $n=n(t, m, a)$. The dimensions of $p$ and $n$ are cells age mat $^{-1}$.

In the cell population, there will be a finite number which are primitive and have not begun differentiation. These can not be characterised in terms of $p$ and $n$ at $m=0$, since the latter are cell densities with respect to $a$ and $m$. The primitive stem cells are characterised by cell densities $p_{0}(t, a)$ and $n_{0}(t, a)$, such that $p_{0} d a$ and $n_{0} d a$ are the numbers of primitive stem cells (with $m=0)$ of age in $(a, a+d a)$.

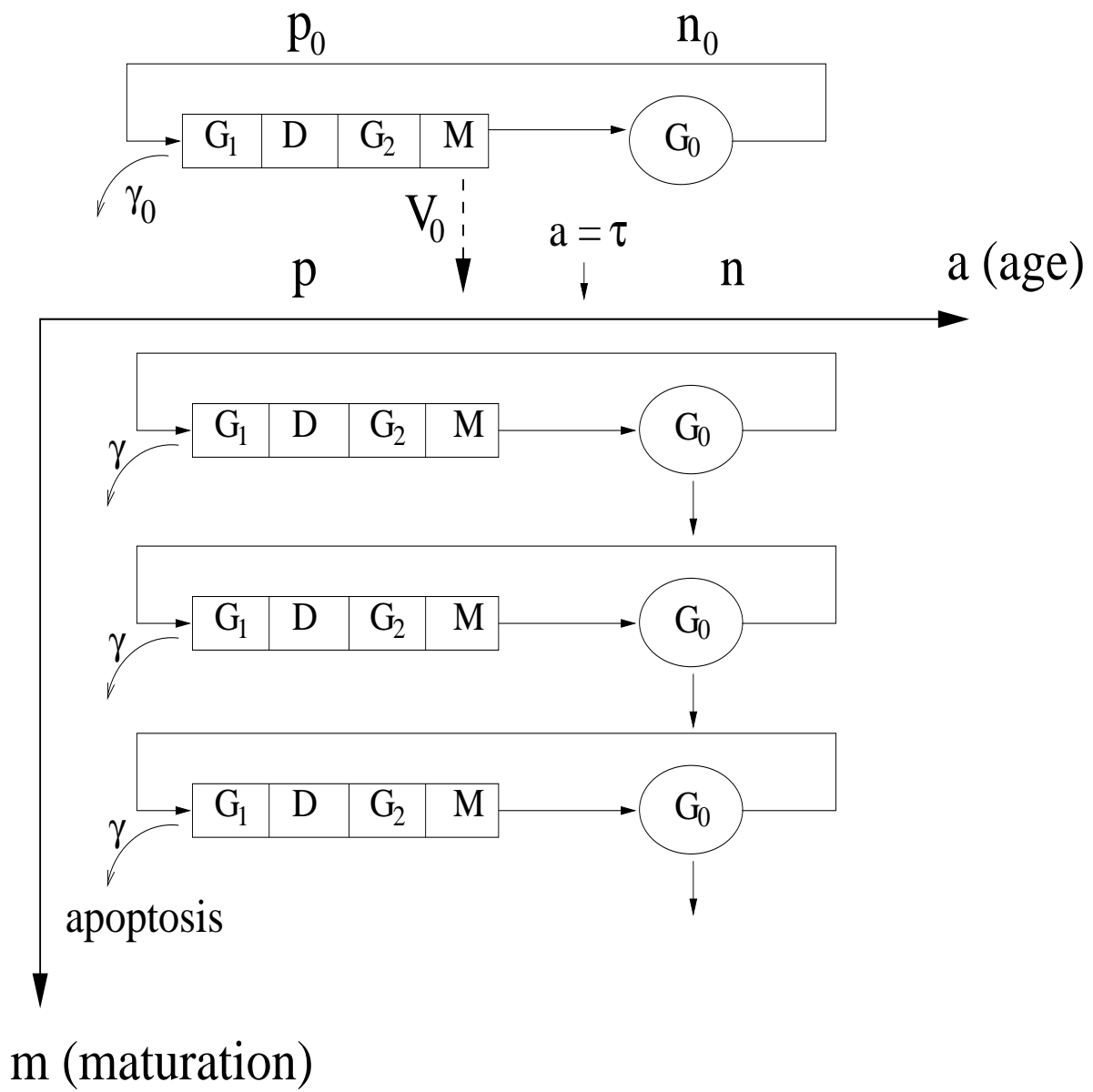

Figure 3: Schematic evolution of cells. Each cell ages as it goes through its cell cycle, before dividing and entering a resting $\left(G_{0}\right)$ phase; at the same time, the cells mature. The time-like variables $a$ (age) and $m$ (maturation) are independent.

The evolution of the system is illustrated schematically in figure 3. We suppose that cell mortality occurs at a rate $\gamma$ (for proliferating cells only), and that cell maturation occurs continuously at a rate $V$ (for both proliferative and resting phases). We suppose that both $\gamma$ and $V$ may depend on maturation stage $m$, but not on $t$. 
Conservation of proliferative cells then implies

$$
\frac{\partial p}{\partial t}+\frac{\partial p}{\partial a}+\frac{\partial(V p)}{\partial m}=-\gamma p
$$

where the units of $V$ are mat $\mathrm{d}^{-1}$ (maturation units per day). We suppose (2.1) applies during a cycle of length $\tau$ (which might depend on $m$ ), thus for $0<a<\tau$; then for $a>\tau$, the cells in the resting phase satisfy the equation

$$
\frac{\partial n}{\partial t}+\frac{\partial n}{\partial a}+\frac{\partial(V n)}{\partial m}=-R n
$$

which differs from (2.1) by the rate of recruitment $R$ back to the proliferative phase; resting cell mortality is taken to be zero. Equation (2.2) applies for $a>\tau$.

At the end of the cell cycle, $a=\tau$, we apply a boundary condition describing the conversion of $p$ to $n$. Mackey and Rudnicki (1994) allow a very general condition, on the basis that cells at maturation $M$ can divide to form cells at maturation $g(M) \leq M$. Specifically, this implies $2 p[t, M, \tau(M)] d M=n[t, g(M), \tau\{g(M)\}] d g$. If we write $m=g(M), M=h(m)$ (so $\left.h=g^{-1}\right)$, then this becomes

$$
n[t, m, \tau(m)]=2 p[t, h(m), \tau\{h(m)\}] h^{\prime}(m),
$$

where $g(m) \leq m$ implies $h(m) \geq m$.

A boundary condition for $p$ at $a=0$ follows from the recruitment condition (the renewal equation)

$$
p(t, m, 0)=R N(t, m),
$$

where we introduce the total resting cell density

$$
N=\int_{\tau}^{\infty} n d a
$$

Now we integrate (2.2) from $a=\tau$ to $a=\infty$, taking $n \rightarrow 0$ as $a \rightarrow \infty$ (which is necessary if there are a finite number of cells). We suppose that $V=V(m)$ is independent of $a$ and $t$, and and $R=R(t, m)$ is independent of $a$. Then

$$
\frac{\partial N}{\partial t}+\frac{\partial(V N)}{\partial m}=-R N+2 p[t, h(m), \tau\{h(m)\}] h^{\prime}(m)
$$

adopting (2.3).

We need to solve (2.1) for $p$. We use the method of characteristics, and begin by applying the recruitment condition (2.4). Specifically, we apply the parametric conditions

$$
t=s, \quad m=\mu, \quad a=0, \quad p=R(s, \mu) N(s, \mu),
$$

valid for $s, \mu>0$; then the characteristic solution is

$$
\begin{aligned}
& a=t-s, \int_{\mu}^{m} \frac{d \rho}{V(\rho)}=t-s \\
& p=R(s, \mu) N(s, \mu) \exp \left[-\int_{s}^{t}\left[\gamma+V^{\prime}(m)\right] d t\right] .
\end{aligned}
$$


Define a function $\nu(m, a)$ by

$$
\int_{\nu}^{m} \frac{d \rho}{V(\rho)}=a
$$

Then $a=t-s, \mu=\nu(m, a)$. Also $d t=d m / V(m)$ on a characteristic, thus for $t>a$ (and also $\nu>0$ ),

$$
p(t, m, a)=R[t-a, \nu(m, a)] N[t-a, \nu(m, a)] \exp \left[-\int_{\nu(m, a)}^{m}\left\{\gamma+V^{\prime}(\rho)\right\} \frac{d \rho}{V(\rho)}\right]
$$

simplifying and putting $a=\tau$, we have

$$
p(t, m, \tau)=R[t-\tau, \nu(m, \tau)] N[t-\tau, \nu(m, \tau)] \exp \left[-\int_{\nu(m, \tau)}^{m} \frac{\gamma d \rho}{V(\rho)}\right] \frac{V[\nu(m, \tau)]}{V(m)}
$$

for $t>\tau$ and $\nu>0$. Finally, (2.6) becomes

$$
\begin{aligned}
& \frac{\partial N}{\partial t}+\frac{\partial}{\partial m}(V N)=- R N \\
&+2 h^{\prime}(m) R[t-\tau, \nu\{h(m), \tau\}] N[t-\tau, \nu\{h(m), \tau\}] \times \\
& \quad \exp \left[-\int_{\nu\{h(m), \tau\}}^{h(m)} \frac{\gamma d \rho}{V(\rho)}\right] \frac{V[\nu\{h(m), \tau\}]}{V[h(m)]} .
\end{aligned}
$$

Note that

$$
\int_{\nu(m, \tau)}^{m} \frac{d \rho}{V(\rho)} \equiv \tau
$$

It is convenient to define a modified maturation variable $\xi$ by

$$
\xi=\int_{0}^{m} \frac{d \rho}{V(\rho)}
$$

$\xi$ has units of time, and indeed it is equal to the elapsed time during maturation. Note that $\nu>0$ if $\xi>\tau$. The lower limit can be chosen for convenience, and allows us to fix $\xi$ at some reference point; here we choose this to be the initial maturation stage (note that this cannot be done if $V(0)=0$ ). Define also

$$
\eta(\xi)=\int_{0}^{h(m)} \frac{d \rho}{V(\rho)}
$$

(note $\eta \geq \xi$ since $h \geq m$ ). Now if

$$
F(m) \equiv f(\xi)
$$

then we find

$$
\begin{aligned}
F[h(m)] & =f(\eta), \\
F[\nu\{h(m), \tau\}] & =f(\eta-\tau) .
\end{aligned}
$$


We change variable from $m$ to $\xi$, and define

$$
\begin{aligned}
v(\xi) & \equiv V(m), \\
M & \equiv N V
\end{aligned}
$$

(note that $M d \xi=N d m$, so that $M$ is cell density in terms of the variable $\xi$; the units of $M$ are cells $\left.\mathrm{d}^{-1}\right)$. After a little manipulation, we find

$$
\frac{\partial M}{\partial t}+\frac{\partial M}{\partial \xi}=-R M+Q
$$

where

$$
Q=2 \eta^{\prime}(\xi) R[t-\tau, \eta-\tau] M[t-\tau, \eta-\tau] \exp \left[-\int_{\eta-\tau}^{\eta} \gamma d \xi\right]
$$

where we write $\gamma, R$ and $M$ as dependent on $\xi$ rather than $m$. This equation applies if $t>\tau$ and $\eta>\tau$.

In order to find the form of the source term for $t<\tau$ or $\eta<\tau$, we must solve the equation (2.1) for $p$ using the initial data from $m=0$ and $t=0$. If, specifically, we have an initial condition

$$
p=p_{I}(m, a) \quad \text { at } \quad t=0,
$$

then after some algebra we find that

$$
Q=2 \eta^{\prime}(\xi) p_{I}[\eta-t, \tau-t] v(\eta-t) \exp \left[-\int_{\eta-t}^{\eta} \gamma d \xi\right], \quad t<\tau, \quad \eta>t .
$$

The definition of $Q$ in $t>\eta$ and $\eta<\tau$ requires consideration of the stem cell evolution, and we now turn to this. Conservation laws for the stem cell densities $p_{0}$ and $n_{0}$ are

$$
\begin{aligned}
\frac{\partial p_{0}}{\partial t}+\frac{\partial p_{0}}{\partial a} & =-\left(\gamma_{0}+V_{0}\right) p_{0} \\
\frac{\partial n_{0}}{\partial t}+\frac{\partial n_{0}}{\partial a} & =-\left(V_{0}+R_{0}\right) n_{0}
\end{aligned}
$$

where $V_{0}$ is the rate of loss of stem cells to maturation, $R_{0}$ is the stem cell recruitment rate from the resting phase, and $\gamma_{0}$ is the mortality rate of stem cells in the proliferative phase. We allow $R_{0}, V_{0}$ and $\gamma_{0}$ to depend on $t$, but we assume they are independent of $a$. Note that $V_{0} \neq 0$, indeed $V_{0} \neq V(0)$, as the units of $V_{0}$ and $V$ are not even the same: $V$ has units of mat $\mathrm{d}^{-1}$, while $V_{0}$ has units of $\mathrm{d}^{-1}$. Note also that $p_{0}$ and $n_{0}$ have units of cells age ${ }^{-1}$ (unlike $p$ and $n$ ).

The primitive loss to maturation must balance the source for $p$ and $n$ at $m=0$, thus

$$
V_{0} p_{0}=\left.(V p)\right|_{m=0}, \quad V_{0} n_{0}=\left.(V n)\right|_{m=0},
$$

and the units are consistent.

Analogously to (2.4) and (2.3), we have

$$
\begin{aligned}
& p_{0}(t, 0)=R_{0}(t) N_{0}(t), \\
& n_{0}(t, \tau)=2 p_{0}(t, \tau),
\end{aligned}
$$


where

$$
N_{0}=\int_{\tau}^{\infty} n_{0} d a
$$

Integration over $a$ now yields

$$
\frac{d N_{0}}{d t}=-V_{0} N_{0}-R_{0} N_{0}+\left.2 p_{0}\right|_{a=\tau}
$$

and

$$
\left.(N V)\right|_{m=0}=N_{0} V_{0}
$$

In order to find $p_{0}$ we must solve

$$
\frac{\partial p_{0}}{\partial t}+\frac{\partial p_{0}}{\partial a}=-\left(\gamma_{0}+V_{0}\right) p_{0}
$$

with parametric initial conditions:

$$
\begin{gathered}
p_{0}=p_{00}(\alpha), \quad a=\alpha>0, \quad t=0, \\
p_{0}=R_{0}(s) N_{0}(s), \quad a=0, \quad t=s>0 .
\end{gathered}
$$

For $t>a$, the solution is

$$
p_{0}=R_{0}(t-a) N_{0}(t-a) \exp \left[-\int_{t-a}^{t}\left[\gamma_{0}\left(t^{\prime}\right)+V_{0}\left(t^{\prime}\right)\right] d t^{\prime}\right],
$$

whereas for $t<a$,

$$
p_{0}=p_{00}(a-t) \exp \left[-\int_{0}^{t}\left[\gamma_{0}\left(t^{\prime}\right)+V_{0}\left(t^{\prime}\right)\right] d t^{\prime}\right] .
$$

Putting $a=\tau$, we find

$\frac{d N_{0}}{d t}=-\left(R_{0}+V_{0}\right) N_{0}+2 R_{0}(t-\tau) N_{0}(t-\tau) \exp \left[-\int_{t-\tau}^{t}\left[\gamma_{0}\left(t^{\prime}\right)+V_{0}\left(t^{\prime}\right)\right] d t^{\prime}\right], \quad t>\tau$,

which prescribes the control system for $N_{0}$, analogously to that of Mackey (1978). For $t<\tau$, the equation for $N_{0}$ involves the initial condition for $p_{0}$, and we can equivalently simply prescribe initial data for $N_{0}$ there.

Finally, we complete the definition of $Q$ in (2.19) by solving (2.1) using the initial data on $m=0$ :

$$
m=0, \quad a=\alpha>0, \quad t=s>0, \quad V(0) p=V_{0}(s) p_{0}(s, \alpha) .
$$

We find

$$
p(t, \xi, a)=\frac{V_{0}(t-\xi) p_{0}(t-\xi, a-\xi)}{v(\xi)} \exp \left[-\int_{0}^{\xi} \gamma d \xi\right], \quad \xi<a, \quad \xi<t,
$$

from which it follows that

$$
Q=2 \eta^{\prime}(\xi) V_{0}(t-\eta) p_{0}[t-\eta, \tau-\eta] \exp \left[-\int_{0}^{\eta} \gamma d \xi\right], \quad t>\eta, \quad \eta<\tau
$$


Along with (2.20) and (2.22), this completes the definition of $Q$ for $t>0, \eta>0$ (and thus $\xi>0)$. In summary,

$$
Q=\left\{\begin{array}{lll}
2 \eta^{\prime}(\xi) R[t-\tau, \eta-\tau] M[t-\tau, \eta-\tau] \exp \left[-\int_{\eta-\tau}^{\eta} \gamma d \xi\right], & t>\tau, & \eta>\tau, \\
2 \eta^{\prime}(\xi) p_{I}[\eta-t, \tau-t] v(\eta-t) \exp \left[-\int_{\eta-t}^{\eta} \gamma d \xi\right], & t<\tau, & \eta>t, \\
2 \eta^{\prime}(\xi) V_{0}(t-\eta) p_{0}[t-\eta, \tau-\eta] \exp \left[-\int_{0}^{\eta} \gamma d \xi\right], & t>\eta, & \eta<\tau .
\end{array}\right.
$$

The two equations (2.33) and (2.19) are coupled through (2.28), which provides the requisite boundary condition for $M$ at $\xi=0$ :

$$
M=V_{0} N_{0} \text { at } \xi=0 .
$$

We see that by an appropriate consideration of the primitive stem cells, we derive a coherent model which does not require $V(0)=0$.

Many authors (for example, see Rey and Mackey (1993) and Dyson et al. (1996)) study the differential equation (2.12) for $N$, under the assumption that $V$ does tend to zero as $m \rightarrow 0$, for example,

$$
V=r m \text {. }
$$

The reasoning behind this is that, if primitive stem cells mature at a finite rate, then such cells will be immediately lost to $m>0$, which makes no physiological sense, because the cell population then inexorably disappears. Only by choosing $V(0)=0$ can we allow primitive stem cells to endure. In the present version of the model, it is also possible to allow $V(0)=0$, for example, (2.39) would then imply

$$
M \rightarrow 0 \text { as } \xi \rightarrow-\infty \text {. }
$$

The sensitivity of the solution to this condition has led to the idea that the system may have unstable and even chaotic solutions (e.g., Crabb et al. (1996)), because of the degeneracy of the equation at $m=0$. Our considerations here suggest that the requirement that $V(0)=0$ is inaccurate, because it does not properly address the biological question of how the primitive stem cells should be described.

\section{Dimensionless model}

How we solve the model depends on the complexity of our assumptions about $\gamma, R$, $\eta$ and $R_{0}$. In the remainder of this paper we will assume $g(m)=m$, thus $\eta=\xi$, and that $\gamma$ and $\gamma_{0}$ are constant. The equation for the maturing cells, (2.19), is

$$
\frac{\partial M}{\partial t}+\frac{\partial M}{\partial \xi}=-R M+Q
$$

and is a hyperbolic delay-partial differential equation. Figure 4 shows the regions where the different definitions of $Q$ apply. In regions II and segment $(a)$ of region 


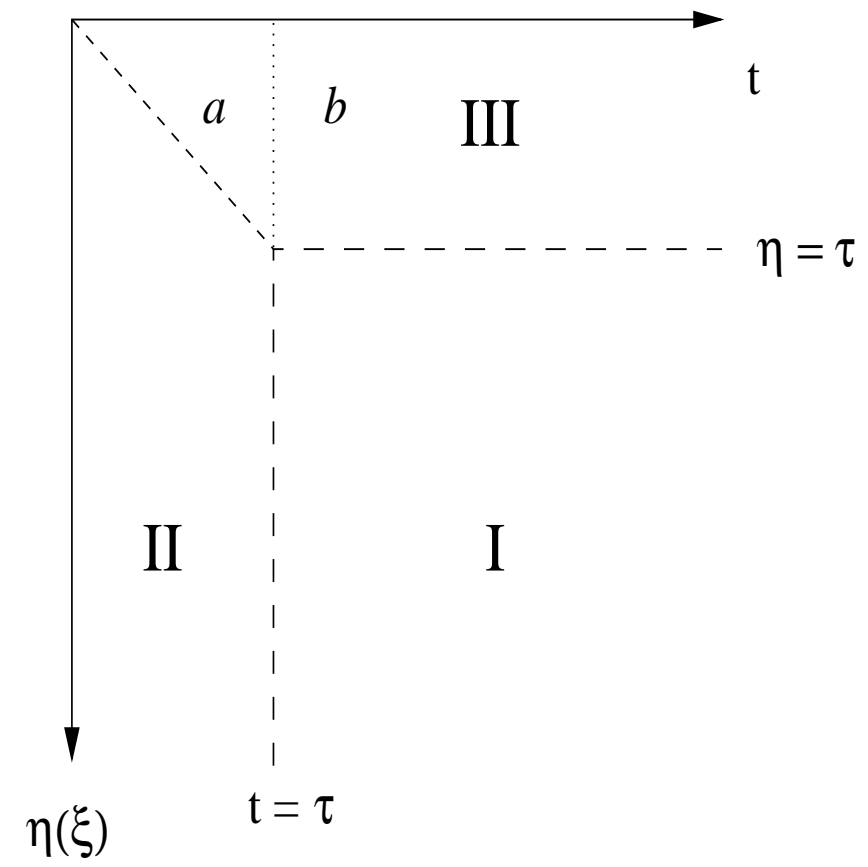

Figure 4: Regions of different definitions of $Q$ in (2.37). Regions I, II and III correspond to the first, second and third definitions of $Q$ and their locations of validity in the $(t, \eta)$ plane. The vertical dotted line in region III (where $Q$ is defined in terms of $\left.p_{0}\right)$ separates the region $(a)$ where (2.32) applies (to the left) from that $(b)$ where (2.31) applies (to the right).

III, that is for $t<\tau$ and all $\eta=\xi>0, Q$ depends on the initial data, either $p_{I}$ (in II) or $p_{00}$ (in $\left.\operatorname{III}(a)\right)$. Thus we may equivalently simply choose instead to prescribe $M$ in $0<t<\tau$, and this we do. In fact, since $\xi$ is finite, the part of the solution which depends on this initial data will wash out of the system in a finite time. It is therefore apparently of little concern.

We therefore confine ourselves to consideration of the definition of $Q$ in regions I and $\operatorname{III}(b)$; with the assumptions we have made, we find that for $t>\tau$,

$Q= \begin{cases}2 e^{-\gamma \tau} R[t-\tau, \xi-\tau] M[t-\tau, \xi-\tau], & \xi>\tau, \\ 2 e^{-\gamma_{0}(\tau-\xi)} e^{-\gamma \xi} \exp \left[-\int_{t-\tau}^{t-\xi} V_{0}\left(t^{\prime}\right) d t^{\prime}\right] V_{0}(t-\xi) R_{0}(t-\tau) N_{0}(t-\tau), & \xi<\tau .\end{cases}$

We thus have to solve (3.1) with (3.2) in $t>\tau$, with the boundary condition (2.38) on $\xi=0$, and prescription of an initial function for $M$ in $0<t<\tau$.

A principal issue of focus is how the recruitment rates $R$ and $R_{0}$ and the committal rate $V_{0}$ depend on $M, N_{0}$ and $\xi$. There is very little to constrain our choice. In what follows, we assume $R_{0}=R_{0}\left(N_{0}\right)$ (stem cell proliferation is controlled by stem cell density). We follow Mackey and Rudnicki (1994) in supposing that $R$ depends on the 
total differentiating cell population $\bar{M}$, where

$$
\bar{M}(t)=\int_{0}^{\xi_{F}} M d \xi
$$

$\xi_{F}$ being the time of final maturation,

$$
\xi_{F}=\int_{0}^{m_{F}} \frac{d \rho}{V(\rho)}
$$

and $m=m_{F}$ at full maturity. We suppose that the rate of committal $V_{0}$ should depend on the peripheral blood cell count, $B$, thus $V_{0}=V_{0}(B)$. A simple model for $B$ is

$$
\frac{d B}{d t}=\left.M\right|_{\xi_{F}}-\gamma_{B} B
$$

where $\gamma_{B}$ is the specific decay rate of the peripheral blood cells, and the source term $\left.M\right|_{\xi_{F}}$ is the delivery rate to the blood from the maturation phase cells. Peripheral control models of similar type have been studied by Bernard et al. (2003). Assumptions of this type are liable to be important in the evolution of diseases such as cyclical neutropenia, which is thought to be due to an instability in the peripheral control of stem cell committal. In addition, it is likely that other controls affect rate of apoptosis, maturation rate, cell cycle time, and so on.

The equation for $N_{0}(2.33)$ now takes the form

$$
\dot{N}=-\left[V_{0}+R_{0}\left(N_{0}\right)\right] N_{0}+2 e^{-\gamma_{0} \tau} R_{0}\left(N_{0 \tau}\right) N_{0 \tau} \exp \left[-\int_{t-\tau}^{t} V_{0}\left[B\left(t^{\prime}\right)\right] d t^{\prime}\right],
$$

where $N_{0 \tau}=N_{0}(t-\tau)$. This is precisely the model of Mackey (1978) if $V_{0}$ is constant, and has been studied by Fowler and Mackey (2002) in the limit

$$
V_{0} \tau \ll 1,
$$

when it is shown that relaxation oscillations will occur for a further parameter $\mu_{0}=$ $\left[2 e^{-\left(\gamma_{0}+V_{0}\right) \tau}-1\right] / V_{0} \tau$ within a certain $O(1)$ range. (Note that in the notation of Fowler and Mackey's model, $\gamma=\gamma_{0}+V_{0}, \delta=V_{0}$.) When such oscillations occur, they will propagate through the maturing cells; however we show in this paper that the resultant amplitude of oscillations of mature blood cells is small unless amplification also occurs during maturation.

We can write (3.2) in abbreviated form as

$$
Q= \begin{cases}2 e^{-\gamma \tau} R\left(\bar{M}_{\tau}\right) M_{\tau, \tau}, & \xi>\tau, \\ 2 e^{-\gamma_{0}(\tau-\xi)} e^{-\gamma \xi} \exp \left[-\int_{t-\tau}^{t-\xi} V_{0}\left[B\left(t^{\prime}\right)\right] d t^{\prime}\right] V_{0}[B(t-\xi)] R_{0}\left(N_{0 \tau}\right) N_{0 \tau}, & \xi<\tau,\end{cases}
$$

where $\bar{M}_{\tau}=\bar{M}(t-\tau)$, and $M_{\tau, \tau}=M(t-\tau, \xi-\tau)$.

We non-dimensionalise the model by following the analysis of Fowler and Mackey (2002), which motivates a choice of scales for the variables as follows:

$$
t, \xi \sim \tau, \quad M \sim M^{*}, \quad N_{0}=N_{0}^{*} S, \quad Q \sim \frac{M^{*}}{\tau}
$$




$$
R_{0}=R_{0}^{*} h_{0}, \quad R=R^{*} h, \quad V_{0}=V_{0}^{*} v_{0}, \quad B \sim \frac{M^{*}}{\gamma_{B}},
$$

where $R_{0}^{*}, R^{*}, V_{0}^{*}, N_{0}^{*}, M^{*}$ are determined by the control functions (so that they are $O(1)$ functions of $O(1)$ variables). For example, Mackey (1978) chooses for $R_{0}$ the Hill function

$$
R_{0}=\frac{R_{0}^{*}}{1+\left(N_{0} / \theta\right)^{n}} .
$$

In this case, we would choose $N_{0}^{*}=\theta$ and $h_{0}$ is the Hill function

$$
h_{0}(S)=\frac{1}{1+S^{n}} .
$$

The dimensionless stem cell equation is

$$
\dot{S}=b_{0}\left[\left(1+\lambda_{0}\right) h_{0}\left(S_{1}\right) S_{1} \exp \left(\varepsilon_{0}\left\{1-\int_{t-1}^{t} v_{0}\left[B\left(t^{\prime}\right)\right] d t^{\prime}\right\}\right)-h_{0} S\right]-\varepsilon_{0} v_{0} S,
$$

where

$$
\varepsilon_{0}=V_{0}^{*} \tau, \quad \lambda_{0}=2 e^{-\left(\gamma_{0}+V_{0}^{*}\right) \tau}-1, \quad b_{0}=R_{0}^{*} \tau .
$$

The dimensionless form of (3.8) is

$$
\frac{\partial M}{\partial t}+\frac{\partial M}{\partial \xi}=-b h(\bar{M}) M+Q
$$

where

$Q=\left\{\begin{array}{l}b(1+\lambda) h\left(\bar{M}_{1}\right) M_{1,1}, \quad \xi>1, \\ \nu b_{0}\left(1+\lambda_{0}\right) e^{-\alpha \xi} \exp \left[\varepsilon_{0}\left\{1-\int_{t-1}^{t-\xi} v_{0}\left[B\left(t^{\prime}\right)\right] d t^{\prime}\right\}\right] v_{0}[B(t-\xi)] h_{0}\left(S_{1}\right) S_{1}, \quad \xi<1,\end{array}\right.$

in which

$$
\nu=\frac{N_{0}^{*} V_{0}^{*}}{M^{*}}, \quad b=R^{*} \tau, \quad \lambda=2 e^{-\gamma \tau}-1, \quad \alpha=\left(\gamma-\gamma_{0}\right) \tau .
$$

This is analogous to the scaling used by Fowler and Mackey (2002). The boundary condition for $M$ is

$$
M=\nu v_{0} S \quad \text { at } \quad \xi=0,
$$

and if

$$
M=M_{f} \quad \text { at } \quad \xi=\xi_{f},
$$

then

$$
\delta \dot{B}=M_{f}-B,
$$

where

$$
\delta=\frac{1}{\gamma_{B} \tau}, \quad \xi_{f}=\frac{\xi_{F}}{\tau} .
$$

This completes the statement of the dimensionless form of the model. 


\section{Parameter values}

The equation (3.12) is exactly that studied by Fowler and Mackey (2002) (if $v_{0} \equiv 1$ ). However, their model can also be interpreted as a 'lumped', or compartmentalised, version of (3.14) for the maturing cells. One way of enabling this is if we make the special assumption that the maturation rate $V \rightarrow 0$ as both $m \rightarrow 0$ and $m \rightarrow m_{F}$, as also assumed by Mackey and Rudnicki (1994). In this case the range of $\xi$ is $(-\infty, \infty)$, and we have $M \rightarrow 0$ at both limits. Then integration of (3.14) over $\xi$ again leads to an equation of the form of (3.12). In the present paper we assume $V$ is finite at $m=m_{F}$, i. e., the mature blood cells are delivered to the bloodstream at a finite rate, and this is then the essential difference between the models with and without maturation.

In estimating the parameters, we follow Fowler and Mackey (2002) in choosing $\tau \sim 2 \mathrm{~d}$, and we suppose that proliferative control is effected at typical rates $R^{*} \sim$ $R_{0}^{*} \sim 2 \mathrm{~d}^{-1}$. We suppose apoptosis rates are of order $\gamma \sim \gamma_{0} \sim 0.2 \mathrm{~d}^{-1}$, and that committal rates are of order $V_{0}^{*} \sim 0.05 \mathrm{~d}^{-1}$, and from these we find

$$
b \sim b_{0} \sim 4, \quad \lambda \sim \lambda_{0} \sim 0.3, \quad \varepsilon_{0} \sim 0.1
$$

The parameter $\alpha$ is not independent of the others, as

$$
\alpha=\varepsilon_{0}+\ln \left(\frac{1+\lambda_{0}}{1+\lambda}\right),
$$

and plausibly $\alpha \approx \varepsilon_{0}$.

The remaining parameters are $\delta, \nu$ and $\xi_{f}$. For $\delta$, we assume a half life $\left(\gamma_{B}^{-1}\right)$ of 7 hours, appropriate for neutrophils (but, for example, certainly not for erythrocytes); then

$$
\delta \sim 0.15
$$

We can get some sense of the size of the remaining parameters $\nu$ and $\xi_{f}$ by considering the nature of stem cells. These are difficult to isolate; indeed it is not yet clear whether genuine stem cells have ever really been isolated. The reason for this is that there are few of them, and maturing cells will typically undergo about (or at least) twenty divisions before emerging as mature blood cells. A typical numerical estimate for the total number of blast cells is $10^{12}$ per $\mathrm{kg}$ body weight, while for stem cells, a corresponding estimate is $10^{6}$ (Bernard et al. (2003), Mackey (2001)). If this is the case, then it successively implies that the parameter $\nu$ in (3.16) is very small $\left(\approx 10^{-6}\right)$, and therefore also that the maturation time is long. Typical estimates of $\xi_{F} \approx 10-20$ days are consistent with values of $\xi_{f} \approx 5-10$, and in fact the small

parameter $\xi_{f}^{-1}$ then plays the rôle corresponding to that of the small parameter $\varepsilon$ in Fowler and Mackey's (2002) analysis.

\section{Steady state}

To elaborate this discussion, we now describe the steady state. For simplicity, we ignore the distinct definition of $Q$ in $\xi<1$, and extend the definition in $\xi>1$ back 
to $\xi=0$. The steady solution of (3.14) and (3.15) is, with $v_{0}=S=1$,

$$
M=\nu e^{s \xi},
$$

where $s$ is the unique positive solution of the pair

$$
\begin{gathered}
s=b h(\bar{M})\left[(1+\lambda) e^{-s}-1\right], \\
\bar{M}=\frac{\nu}{s}\left(e^{s \xi_{f}}-1\right) .
\end{gathered}
$$

(Uniqueness follows from the fact that $\bar{M}$ is monotonically increasing with $s$, hence $h(\bar{M})$ is monotonically decreasing with $s$, hence $b h(\bar{M})\left[(1+\lambda) e^{-s}-1\right]$ is monotonically decreasing with $s$, while evidently $s$ is increasing.) We can see that $s<\ln (1+\lambda)$, and $s$ will be close to this value if $b$ is large. Note also that by choosing $\lambda_{0}$ and $\varepsilon_{0}$ to have certain specific values which depend on $\lambda, b, \nu$ and $\xi_{f}$, this solution consistently extends back to $\xi=0$, even allowing for the distinct definition of $Q$ in $\xi<1$.

Numerical solutions do confirm the exponential variation of $M$ with $\xi$. In general, it is found that $M$ decreases for $0<\xi<1$, before subsequently increasing.

\section{Periodic solutions}

We are interested in finding whether periodic solutions can occur. There are three different controllers in the model, and thus three different ways in which oscillations can occur: these are described below. We define a default reference set of parameters, and these are given in table 1. They are those suggested by independent estimate, except that we take $\nu=10^{-2}$ rather than $10^{-6}$. This is partly for numerical expediency, as smaller values of $\nu$ require larger $\xi_{f}$ and thus longer computation times, and also because the value of $\nu$ is not well constrained.

\begin{tabular}{|c|c|}
\hline Symbol & Typical value \\
\hline$n$ & 3 \\
$\varepsilon_{0}$ & 0.1 \\
$\lambda_{0}$ & 0.3 \\
$b_{0}$ & 4 \\
$\nu$ & $10^{-2}$ \\
$b$ & 4 \\
$\lambda$ & 0.3 \\
$\xi_{f}$ & 5 \\
$\delta$ & 0.2 \\
$v^{*}$ & 1 \\
$v^{\prime}$ & 1 \\
\hline
\end{tabular}

Table 1: Default parameter values. 
We choose the Hill function controller (3.11) for both $h$ and $h_{0}$, thus

$$
h(\bar{M})=\frac{1}{1+\bar{M}^{n}}, \quad h_{0}(S)=\frac{1}{1+S^{n}},
$$

and we take the peripheral controller $v_{0}$ to have the form

$$
v_{0}=\left[v^{*}-v^{\prime} B\right]_{+},
$$

with default values of the amplitude and slope parameters to be $v^{*}=v^{\prime}=1$. The choice of a threshold in (4.2) is motivated by the observation that neutrophil populations can dwindle to zero in cyclical neutropenia, which would appear to require zero production for sufficiently high blood cell counts.

With this choice of the controller functions and using the default parameters, the steady state is stable. Instabilities arising from parameter variations are described below.

\section{Numerical method}

We have to solve the two ordinary differential equations (3.12) and (3.19), and the partial differential equation (3.14). The solution is complicated by the presence of the integral in (3.12). We define

$$
U=S \exp \left[\varepsilon_{0} \int^{t} v_{0}\left[B\left(t^{\prime}\right)\right] d t^{\prime}\right],
$$

and then $S$ and $U$ satisfy the pair of equations

$$
\begin{gathered}
\dot{S}=S\left[\frac{\dot{U}}{U}-\varepsilon_{0} v_{0}\right], \\
\dot{U}=b_{0}\left[(1+\lambda) e^{\alpha} h_{0}\left(S_{1}\right) U_{1}-h_{0}(S) U\right] .
\end{gathered}
$$

On the assumption that $S$ remains bounded, $U$ grows exponentially as $U \sim \exp \left(\bar{v}_{0} t\right)$, where $\bar{v}_{0}$ is the mean of $v_{0}$. This is likely to cause difficulty in numerical solutions, and this can be reduced by using the algebraically growing function $W=\ln U$, whence

$$
\begin{gathered}
\dot{S}=S\left[\dot{W}-\varepsilon_{0} v_{0}\right], \\
\dot{W}=b_{0}\left[(1+\lambda) h_{0}\left(S_{1}\right) e^{\alpha+W_{1}-W}-h_{0}(S)\right] .
\end{gathered}
$$

In our numerical solutions, we solve (4.5) using the second order accurate improved Euler method, and we similarly solve (3.14) along the characteristics $\xi-t=\eta$, on which the function $Q$ takes the form

$$
Q=\left\{\begin{array}{l}
b(1+\lambda) h\left(\bar{M}_{1}\right) M_{1}, \quad \xi>1, \\
b_{0}(1+\lambda) e^{\alpha(1-\xi)} M_{0}(\eta) h_{0}\left(S_{1}\right) \exp \left[W_{1}-W(\eta)\right], \quad \xi<1,
\end{array}\right.
$$

where $M_{0}(\eta)=M(\eta, 0)$ and $M_{1}=M_{1,1}$, i. e., $M$ delayed by one along the characteristic.

Accurate solutions are obtained with a time step $\Delta t=\Delta \xi=0.05$, and these were checked aginst values $\Delta t=\Delta \xi=0.02$ (which are used to give the figures). 


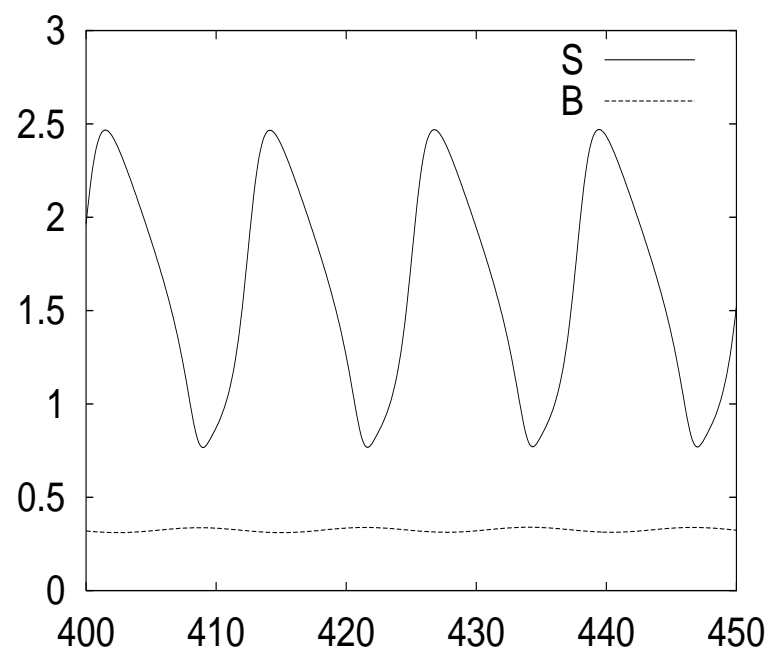

Figure 5: Default parameter values, except that $\lambda_{0}=0.05$. Stem cell oscillations are induced, without any significant effect on blood cells.

\section{Stem cell oscillations}

Oscillations in the primitive stem cell population will occur for a finite range of the parameter $\lambda_{0} / \varepsilon_{0}$, as described by Fowler and Mackey (2002), when $v_{0}=1$. For the default values of $b_{0}=4, n=3$, the approximate range of instability is $0.5 \varepsilon_{0} \lesssim \lambda_{0} \lesssim 1.5 \varepsilon_{0}$, and this is modified in an obvious way when the peripheral controller alters the value of $v_{0}$. Figure 5 shows the oscillations which occur in the stem cell population when $\lambda_{0}$ is reduced to 0.05 . It is an interesting fact that these oscillations are hardly manifested in the blood cell population. The apparent reason for this is that the small value of $\nu$ means that oscillations in $M_{0}$ are small, and therefore also in $M_{f}$, because small perturbations propagate stably down the maturation axis. The blood cell population is therefore stable, and $B \approx M_{f}$.

\section{Proliferation-controlled oscillations}

We use the term proliferation-controlled oscillations to refer to oscillations induced by destabilisation of the proliferative feedback control function $h(\bar{M})$. If we compare the stem cell model (3.12) (with $v_{0}=1$ )

$$
\dot{S}=b_{0}\left[\left(1+\lambda_{0}\right) h_{0}\left(S_{1}\right) S_{1}-h_{0}(S) S\right]-\varepsilon_{0} S
$$

with the blast cell model (along the characteristics)

$$
\dot{M}=b\left[(1+\lambda) h\left(\bar{M}_{1}\right) M_{1,1}-h(\bar{M}) M\right]
$$

it is not difficult to sense that modification of the parameters $b$ or $\lambda$ may cause the blast maturation to proceed unstably. 


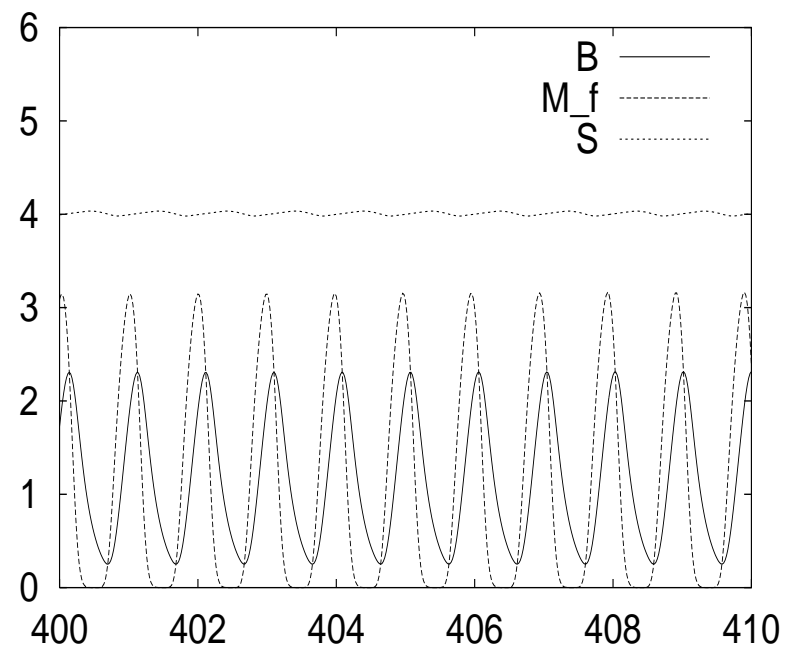

Figure 6: Proliferatively controlled oscillations due to increased proliferation. Default parameter values are used, except that $\lambda=0.6$.

This is what we find if $\lambda$ is increased to 0.6 , and the consequent oscillations are shown in figure 6. The steady exponential proliferation of blast cells is unstable, and this causes oscillations to occur in the maturation profile, and these oscillations propagate along the characteristics, as shown in figure 7.

The oscillations have period equal to the cell cycling time, equal to one in our scaled model. A partial understanding of these oscillations is afforded by the observation that if $h$ is constant and $M$ is periodic with period $2 \pi / \omega$, then (4.8) admits a solution

$$
M=\sum_{p, q} c_{p q} e^{\sigma_{q} \xi+i p \omega(t-\xi)}
$$

provided $\sigma_{q}$ satisfies

$$
\sigma=-A-G e^{-\sigma}
$$

where

$$
A=b h, \quad G=-b h(1+\lambda) .
$$

Since $\lambda>0$, we have $G+A<0$, and it is straightforward to show that there is always a single positive root, which can be labelled with $q=0$. The others are complex (conjugates), and are labelled with increasing frequency as $q= \pm 1, \pm 2$, etc. Consideration of these complex roots then shows that for small $|G|, \operatorname{Re} \sigma_{q}<0$, so that the effect of the oscillations dies away as the cells mature; this is what happens in figure 5. However, for larger $|G|$, Re $\sigma_{q}>0$, and the oscillations grow in amplitude as the cells mature. This causes $\bar{M}$ to fluctuate, and thus also $h$, presumably entraining the period of the oscillations to that of the delay. This description is consistent with what is seen in figure 7 (see also figure 8). An approximate criterion for growth of 


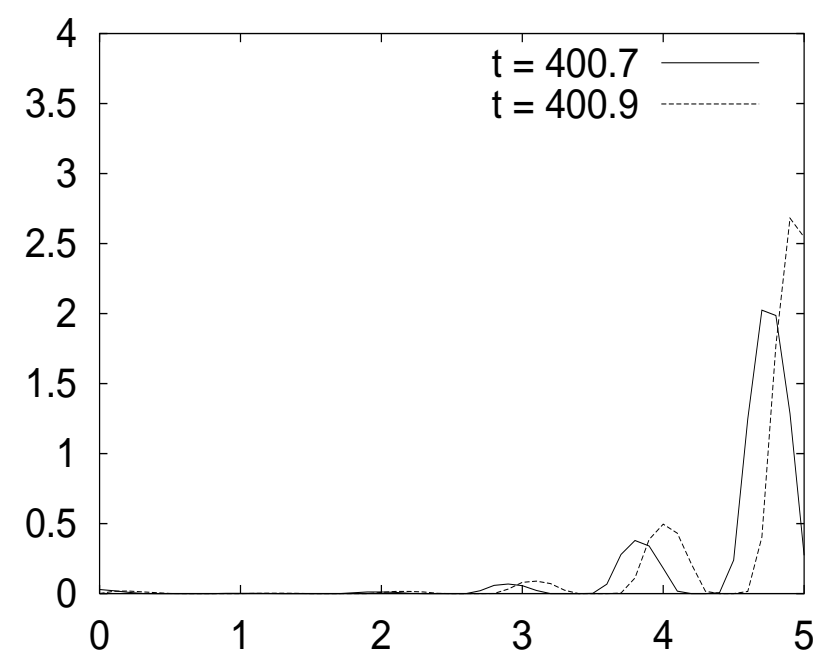

Figure 7: Two snapshots of the maturation profile for the numerical solution in figure 6. An exponentially growing travelling wave propagates down the maturation axis.

periodic perturbations with $\xi$ is when $G<-\left[A^{2}+\pi^{2}\right]^{1 / 2}$, i. e.,

$$
\lambda \gtrsim\left[1+\left(\frac{\pi}{b h}\right)^{2}\right]^{1 / 2}-1 .
$$

\section{Differentiation-controlled oscillations}

The final kind of oscillation that we see is induced by the peripheral control of stem cell committal through the function $v_{0}(B)$. These are essentially delay induced oscillations, where now the delay involved is the maturation time. Because we suppose maturation time is large, these are long period oscillations. They can be caused by increasing the sensitivity of the peripheral controller, as shown in figure 9

To understand the origin of these oscillations, let us suppose that $\xi_{f} \gg 1$, or $\xi_{F} \gg \tau$, meaning that the maturation time is significantly longer than the cell cycle time, or equivalently that there are a large number of generations in the cell lineage. Let us define

$$
\varepsilon=\frac{1}{\xi_{m}},
$$

and the slow time and maturation scales

$$
T=\varepsilon t, \quad X=\varepsilon \xi .
$$

We also define $\mu$ via

$$
\lambda=\varepsilon \mu,
$$

and suppose that $\mu=O(1)$. Essentially we are revisiting the relaxation oscillation analysis of Fowler and Mackey (2002). The partial differential equation for $M$ takes 


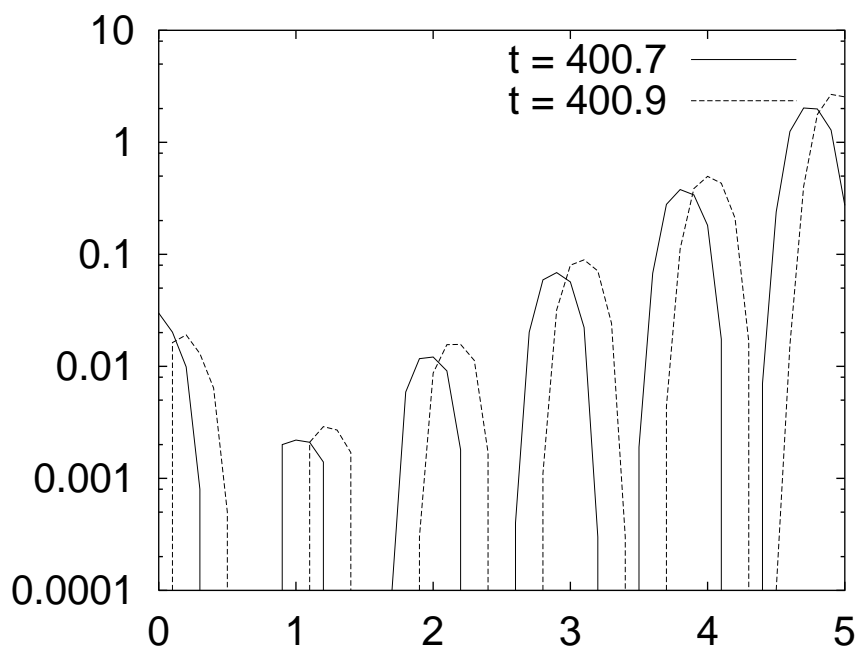

Figure 8: The same graph as in figure [7, except that a logarithmic scale is used. The exponential increase with $\xi$ is clearly seen.

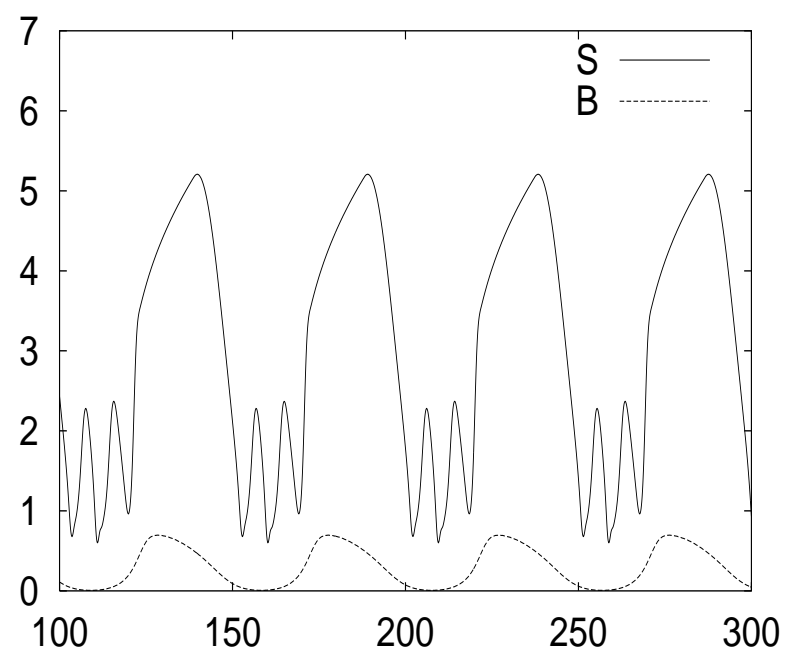

Figure 9: Differentiation-controlled oscillations due to enhancement of the peripheral controller function. Default parameters are used, except that $v^{*}=v^{\prime}=2$. 
the form

$$
\frac{\partial M}{\partial T}+\frac{\partial M}{\partial X}=\frac{b\left[h_{\varepsilon, \varepsilon} M_{\varepsilon, \varepsilon}-h M\right]}{\varepsilon}+\mu b h_{\varepsilon, \varepsilon} M_{\varepsilon, \varepsilon}
$$

and expanding in a Taylor series, we have

$$
\frac{\partial[(1+b h) M]}{\partial T}+\frac{\partial[(1+b h) M]}{\partial X} \approx \mu b h M
$$

with the boundary condition (taking $S=1$ )

$$
M=\nu v_{0}(B) \quad \text { at } \quad X=0 .
$$

If we suppose $h$ is constant (it is not, but it is not the dependence of $h$ on $\bar{M}$ which causes the oscillations), then the solution of this is

$$
M=\nu v_{0}[B(T-X)] \exp \left[\frac{\mu b h X}{1+b h}\right],
$$

and the cell efflux at $X=1\left(\xi=\xi_{f}\right)$ is

$$
M(1)=\nu a v_{0}[B(T-1)],
$$

where the amplification factor $a$ is

$$
a=\exp \left[\frac{\mu b h}{1+b h}\right] .
$$

Therefore the blood cell conservation law (3.19) becomes the delay recruitment model

$$
\varepsilon \delta \frac{d B}{d T}=\nu a v_{0}\left(B_{1}\right)-B
$$

This is a standard delay recruitment equation with a unique steady state. Oscillations will occur as a consequence of instability if there are solutions $\sigma$ of (4.10), i. e.,

$$
\sigma=-A-G e^{-\sigma}
$$

with positive real part. The values of $A$ and $G$ are

$$
A=\frac{1}{\varepsilon \delta}, \quad G=\frac{\nu a\left|v_{0}^{\prime}\right|}{\varepsilon \delta}
$$

The equation (4.23) is well understood, see for example Mackey (1978) or Murray (2002, pp. 23-26). It is a transcendental equation with an infinite number of complex roots which accumulate at the essential singularity at $\sigma=-\infty$. It follows that the set of $\operatorname{Re} \sigma$ is bounded above. Consequently, there is an instability criterion which determines when all the roots $\sigma$ have negative real part, and this is indicated in figure 10. The three curves in the figure are given by $G=-A, G=\exp [-(1+A)]$, and the Hopf bifurcation curve $G=G_{0}(A)$, which is given parametrically by

$$
A=-\frac{\Omega}{\tan \Omega}, \quad G_{0}=\frac{\Omega}{\sin \Omega},
$$




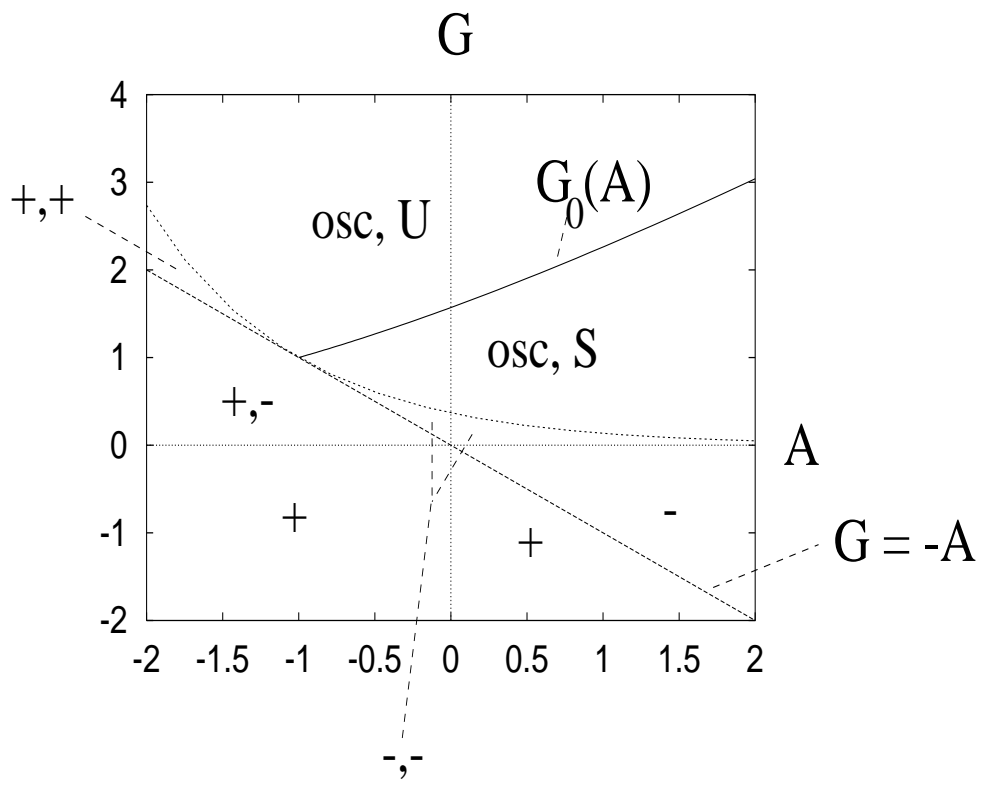

Figure 10: Stability map for (4.23). The plus and minus signs indicate the sign of real values of the growth rate $\sigma$, when these exist. A Hopf bifurcation occurs as $G$ increases through $G_{0}(A)$, and $G_{0} \approx A$ for large $A$.

where $\Omega \in[0, \pi]$. Since $G$ and $A$ are positive, oscillatory instability occurs precisely if $G>G_{0}(A)$. Since $G_{0} \sim A$ as $A \rightarrow \infty$, the instability criterion for large $A$ is simply $G \gtrsim A$, i. e.,

$$
\nu\left|v_{0}^{\prime}\right| \exp \left[\frac{\mu b h}{1+b h}\right]>1 .
$$

Instability is promoted by increasing $\left|v_{0}^{\prime}\right|$, for example, as indicated in figure 9 ,

\section{Conclusions}

In this paper we have studied the onset of oscillations in a model of blood cell production which includes a description of cell cycling and proliferation, and also of differentiation and maturation. The model formulation extends the work of previous authors by correcting an apparent inconsistency in the description of the primitive stem cell population, and also by including the simultaneous control of stem cell proliferation, stem cell committal, and blast cell proliferation. All three controls can cause oscillations for appropriate values of control parameters.

Previous results concerning stem cell oscillations are reproduced (see figure 5) but these oscillations are harder to obtain when the parameter $\nu$ is small, and in addition they hardly affect the mature blood cell population, without additional destabilisation of the blast cell proliferation. The reason for this is that an $O(1)$ oscillation in the stem cell population only causes an $O(\nu)$ oscillation in the blast cell committal rate, and this amplitude propagates through the differentiating cells. Thus one consequence of 


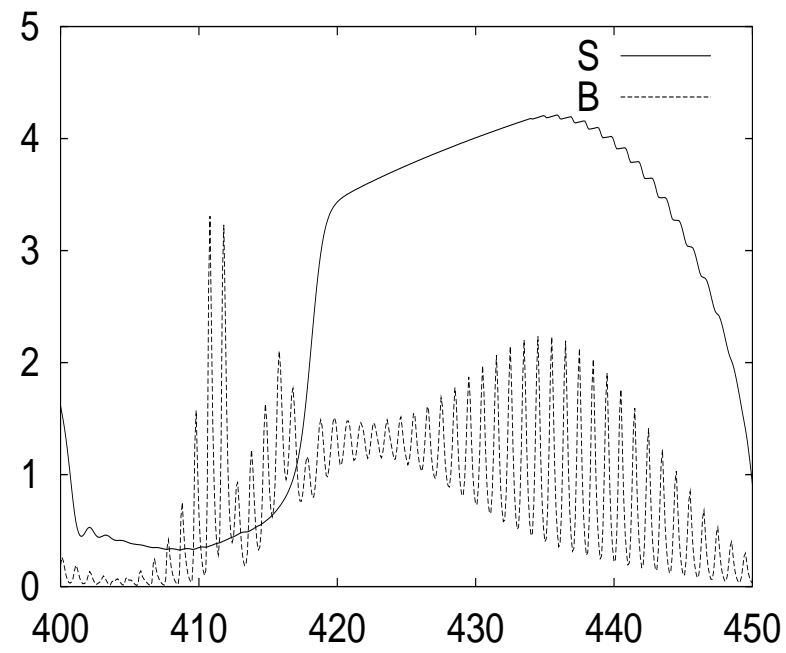

Figure 11: The effect of switching on all three instability mechanisms. Default parameters are used, except that $v^{*}=v^{\prime}=2, \lambda_{0}=0.05$, and $\lambda=0.6$.

stem cell paucity is that any instability in the stem cell population itself is hardly manifested in the blood cell production. From the point of view of survival and control, this is of course a positive result.

Instability in the proliferation of blast cells due to enhancement of the proliferative controller $h(\bar{M})$ causes oscillations which propagate down the maturation axis, and are amplified as they progress. The result of this is shown in figures 6, 7 and 8 . The oscillations have a period equal to the cell cycling time. The mechanism for these oscillations appears to be a destabilisation of the maturing cell amplification, together with a type of resonance which ties the period to the delay.

The final kind of oscillation is induced by enhanced peripheral control, as seen in figure 9. Stem cell paucity implies that $\nu \ll 1$, and consequently that $\xi_{f} \gg 1$, and thus that the oscillation period (controlled by the delay $\xi_{f}$ ) is long. This allows an approximate reduction of the partial differential delay equation to a simple first order differential delay equation, which is simply analysed. In particular, if a threshold form of peripheral controller is used, blood cell counts can decrease to zero, as can be the case in cyclical neutropenia.

Finally, and as shown in figure 11, a combination of all three destabilising mechanisms can lead to oscillations which operate on both the slow, peripherally controlled time scale and the fast, proliferatively controlled one. We consider this observation to be a possible explanation of the apparent fact in figure 2 that both reticulocytes and platelets appear to oscillate on a fast as well as a slow time scale. Further study of this behaviour requires the extension of this model to accommodate multiple cell lineages. 


\section{References}

Bernard, S., J. Bélair and M. C. Mackey 2003 Oscillations in cyclical neutropenia: new evidence based on mathematical modeling. J. Theor. Biol. 223, 283-298.

Chikkappa, G., G. Borner, H. Burlington, A. D. Chanana, E. P. Cronkite, S. Öhl, M. Pavelec and J.S. Robertson 1976 Periodic oscillation of blood leukocytes, platelets, and reticulocytes in a patient with chronic myelocytic leukemia. Blood 47, 1023-1030.

Crabb, R., M. C. Mackey and A. D. Rey 1996 Propagating fronts, chaos and multistability in a cell replication model. Chaos 6, 477-492.

Dale, D. C. and W. P. Hammond IV 1988 Cyclical neutropenia: a clinical review. Blood Revs. 2, 178-185.

Dyson, J., R. Villella-Bresson and G.F. Webb 1996 A singular transport equation modelling a proliferating maturity structured cell population. Can. Appl. Math. Quart. 4, 65-95.

Fokas, A. S., J. B. Keller and B. D. Clarkson 1991 Mathematical model of granulocytopoiesis and chronic myelogenous leukaemia. Cancer Research 51, 2084-2091.

Fortin, P. and M. C. Mackey 1999 Periodic chronic myelogenous leukemia: Spectral analysis of blood cell counts and etiological implications. Br. J. Haematol., 104, 336-345.

Fowler, A.C. and M.C. Mackey 2002 Relaxation oscillations in a class of delay differential equations. SIAM J. Appl. Math. 63, 299-323.

Guerry IV, D., D. C. Dale, M. Omine, S. Perry and S. M. Wolff 1973 Periodic hematopoiesis in human cyclic neutropenia. J. Clin. Investig. 52, 3,220-3,230.

Haurie, C., D. C. Dale and M. C. Mackey 1998 Cyclical neutropenia and other periodic hematological disorders: a review of mechanisms and mathematical models. Blood 92, 2629-2640.

Mackey, M. C. 2001 Cell kinetic status of haematopoietic stem cells. Cell. Prolif. 34, 71-83.

Mackey, M.C. 1978 A unified hypothesis for the origin of aplastic anaemia and periodic haematopoesis. Blood 51, 941-956.

Mackey, M. C. and R. Rudnicki 1994 Global stability in a delayed partial differential equation describing cellular replication. J. Math. Biol. 33, 89-109.

Mackey, M.C. and R. Rudnicki 1999 A new criterion for the global stability of simultaneous cell replication and maturation processes. J. Math. Biol. 38, 195219 . 
Murray, J.D. 2002 Mathematical biology. I: an introduction. Springer-Verlag, Berlin.

Rey, A. D. and M. C. Mackey 1993 Multistability and boundary layer development in a transport equation with delayed arguments. Can. Appl. Math. Quart. 1, $61-81$. 